LA-UR-01-6194

Approved for public release; distribution is unlimited.

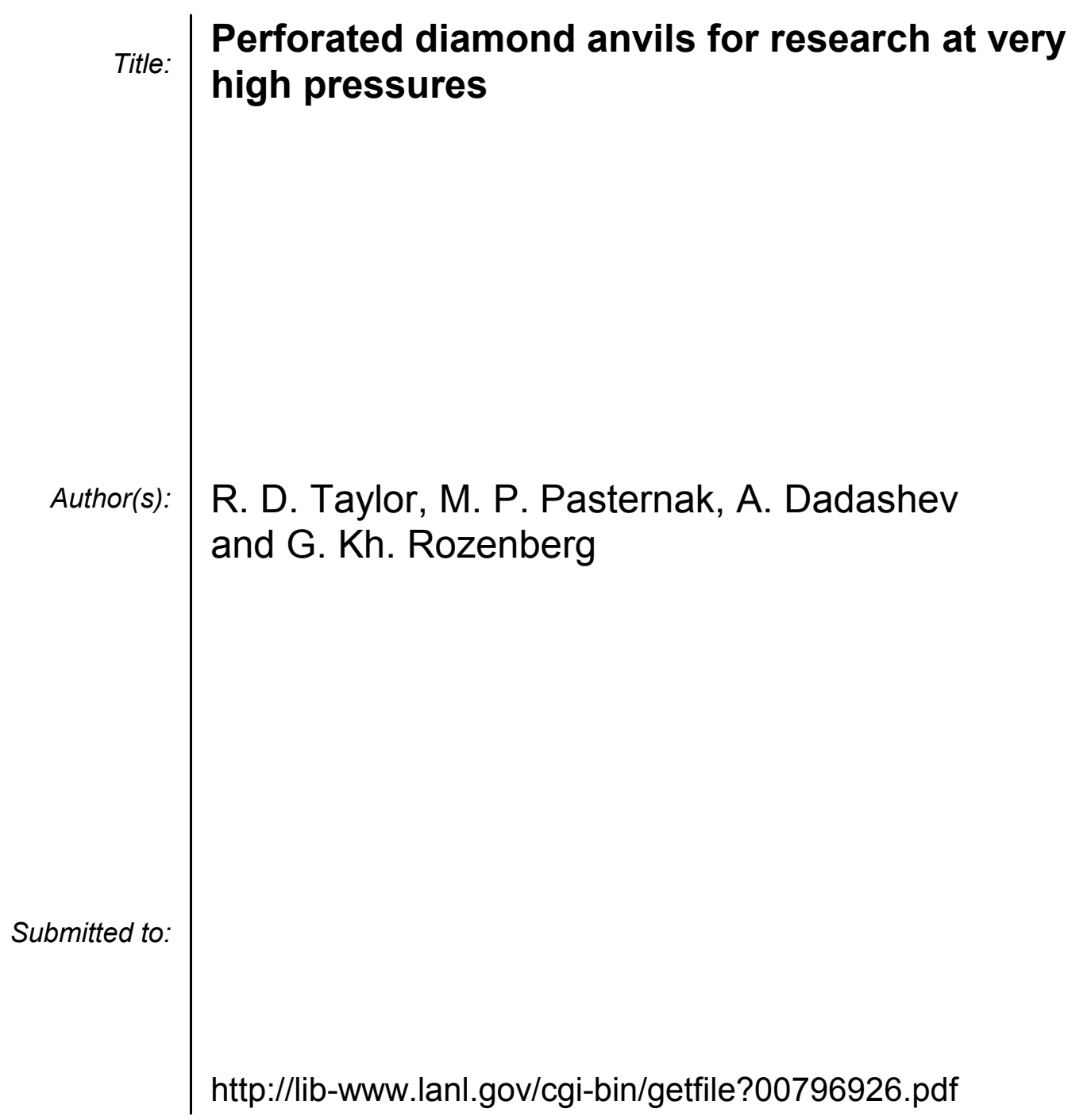

Los Alamos National Laboratory, an affirmative action/equal opportunity employer, is operated by the University of California for the U.S. Department of Energy under contract W-7405-ENG-36. By acceptance of this article, the publisher recognizes that the U.S. Government retains a nonexclusive, royaltyfree license to publish or reproduce the published form of this contribution, or to allow others to do so, for U.S. Government purposes. Los Alamos National Laboratory requests that the publisher identify this article as work performed under the auspices of the U.S. Department of Energy. Los Alamos National Laboratory strongly supports academic freedom and a researcher's right to publish; as an institution, however, the Laboratory does not endorse the viewpoint of a publication or guarantee its technical correctness. 


\title{
Perforated diamond anvils for research at very high pressures
}

\author{
R. D. Taylor ${ }^{\mathrm{a},{ }^{*}}$, M. P. Pasternak ${ }^{\mathrm{a}, \mathrm{b}}$, A. Dadashev ${ }^{\mathrm{b}}$ and G. Kh. Rozenberg ${ }^{\mathrm{b}}$ \\ ${ }^{a}$ MST-10, MS-K764, Los Alamos National Laboratory, Los Alamos NM 87545, USA. \\ ${ }^{b}$ School of Physics and Astronomy, Tel Aviv University, 69978 Tel Aviv, ISRAEL.
}

\begin{abstract}
Diamond anvil cells allow static pressures on small samples up to a few hundred GPa. The anvils also serve as an optical window to the sample. Although the anvils are rather transparent for most types of radiation, the absorption of $\gamma$ - or soft $\mathrm{x}$-rays in a few $\mathrm{mm}$ of diamond can prevent or seriously degrade the quality of the Mössbauer spectrum. Using smaller (thinner) diamonds helps, but then the ultimate pressure suffers. Several schemes are proposed and tested to reduce the pathway (absorption) by drilling away most of the anvil in the axial path, yet retaining its strength and stability.

Key words: High pressures, Diamond anvil cells, Mössbauer spectroscopy
\end{abstract}

\section{INTRODUCTION}

Most of the recent progress in high pressure science may be attributable to the development and use of the diamond anvil cell (DAC) $[1,2]$. With a table to culet tip area ratio of $\sim 1000$, pressures up to over $200 \mathrm{GPa}$ can be generated by an opposing diamond anvil pair held in a simple structure that also supplies the modest closure force. Figure 1 is a generic DAC showing the backing plates, anvils, and a gasket made of a high- $Z$ hard metal into which a sample cavity is formed. A hydrostatic-pressure medium and ruby chips, for in situ determination of $\mathrm{P}$, are added to the sample [1]. The select $\sim 1 / 3$ carat, brilliant-cut natural diamonds modified with 16-sided faceting and a beveled anvil tip that are used to reach these high pressures, are about 2.6-mm thick. Attenuation of a pair of such anvils can seriously degrade Mössbauer experiments, especially for energies below $\sim 20 \mathrm{keV}$. Experience 
has showed that smaller, and therefore thinner diamonds break before reaching 20-30 GPa. An alternative is to remove much of the diamond in the path through drilling or perforation.

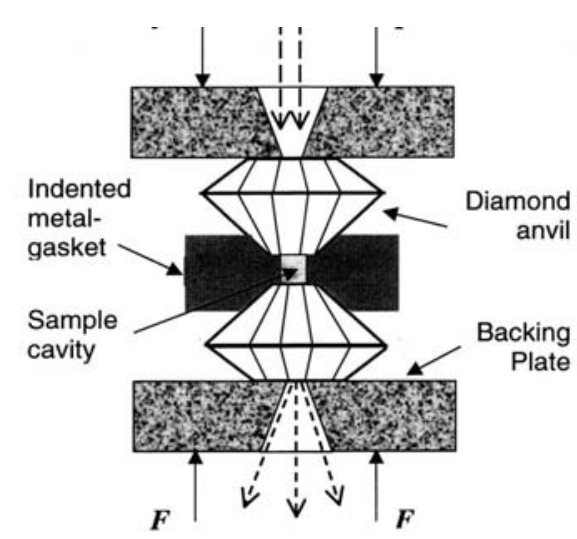

Figure 1. Essential components of a diamond anvil cell. The closure and force mechanism vary for different DACs models.

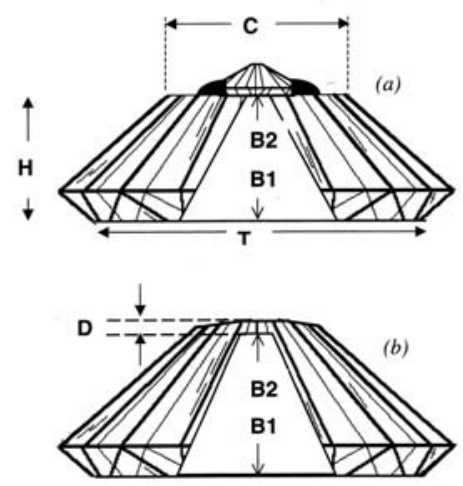

Figure 2. (a) Perforated diamond backing plate with miniature anvil attached. (b) Partially perforated diamond anvil.

This approach has been used by Bassett et al.[3] for soft $\mathrm{x}$ rays in DAC's up to $0.8 \mathrm{GPa}$. We also show that by using fully penetrated diamonds as a backing plate support for miniature (1/100 carat) diamond anvils, one can achieve pressures of $100 \mathrm{GPa}$, and furthermore, this combination is sturdier than the equivalent, undrilled, conventional anvil.

Many MS (mostly ${ }^{57} \mathrm{Fe},{ }^{119} \mathrm{Sn},{ }^{129} \mathrm{I}$, and ${ }^{151} \mathrm{Eu}$ ) and XRD experiments [4] have been carried out despite the attenuation of a pair of 0.3 carat anvils; however we shall show that for ${ }^{57} \mathrm{Fe} \mathrm{MS}$ dramatic improvements are possible using perforated diamonds. The transmission of a pair of regular 0.3 carat ( 5 $\mathrm{mm}$ ) for $14.4 \mathrm{keV}$ is $\sim 22 \%$. With a combination of perforated diamonds and a pair of miniature anvils of $1.6 \mathrm{~mm}$ this becomes $62 \%$. A more detailed report of this work and its applications to other spectroscopies has recently been published [5].

\section{EXPERIMENTAL}

Experiments were conducted to test the performance of the fully perforated anvils, designated as a diamond backing plate (DBP), combined with the miniature anvils (MA), and the partially perforated anvil (PPA), up to $100 \mathrm{GPa}$. Four 0.3-, three 0.012-, and one 0.1-carat diamond anvils of the modified-brilliant-cut design were obtained from D'ANVILS for the present work. All anvils were selected from gem quality stock with minimal internal flaws. Three of the 0.3 carat anvils, $1.6-\mathrm{mm}$ high $(\mathrm{H}), 3.7-\mathrm{mm}$ diam. table 
(T), and 1.8-mm diam. culets $(\mathrm{C})$, were chosen for laser drilling. The conical holes, polished throughout, were 1-, 1.5-, and 2-mm diam. at the table (B1) and $0.3-\mathrm{mm}$ diam. at the culet face (B2) (see figure 2a). The fourth 0.3 carat anvil, with $\mathrm{H}=2.2 \mathrm{~mm}, \mathrm{~T}=3.7 \mathrm{~mm}$, and $\mathrm{C}=1.8 \mathrm{~mm}$ beveled to give a 0.2 $\mathrm{mm}$ flat was partially drilled, leaving a residual wall of $0.5-\mathrm{mm}$ thickness under the culet with $2.0-\mathrm{mm} \mathrm{B} 1$ diam. and $0.3-\mathrm{mm} \mathrm{B} 2$ diam. (see figure $2 \mathrm{~b}$ ). The specifications of the three miniature anvils were: $\mathrm{H}=0.8 \mathrm{~mm}, \mathrm{~T}=1.1$ $\mathrm{mm}$, and $\mathrm{C}=0.2$ or $0.3 \mathrm{~mm}$.

Various combinations of regular anvil, PPA, and DBP+MA were tested using a miniature piston-cylinder DAC that has a flat and a hemispheric tungsten carbide backing plate allowing final cell alignment. The MA was centered and epoxied to the DBP. Rhenium gaskets, preindented to $25 \mu \mathrm{m}$ and drilled to form a sample space half the culet diam., were filled with ruby chips and liquid Ar for pressure tests and with Fe foil for MS studies.

\section{RESULTS}

Except for one MA, all components survived the $100 \mathrm{GPa}$ tests. Of primary interest here, we show the enhanced MS of ${ }^{57} \mathrm{Fe}$ in iron in a DAC with a PPA and a culet matching $\mathrm{DBP}+\mathrm{MA}$ anvil. The latter is required in order to view the sample and its ruby; as the hole in the PPA is not of optical quality. These MS results (figure 3a) are compared with that using regular 0.3 carat anvils (figure $3 \mathrm{~b}$ ). The large improvement reflects improved count rate and improved "good/bad" counts in the $14.4 \mathrm{keV}$ window.

It was also found that wider effective apertures were possible with the $\mathrm{DBP}+\mathrm{MA}$ arrangement than with the regular anvils. DAC's with regular 0.3 -carat anvils mounted on WC backing plates with an aperture greater than $1.2 \mathrm{~mm}$ failed to reach $40 \mathrm{GPa}$. In contrast $100 \mathrm{GPa}$ pressures were reached with 1.0-2.0-mm conical apertures. Wide exit apertures are generally important, especially with MS and XRD.

Following this technological development high pressure MS with lowenergy $\gamma$-rays such as ${ }^{181} \mathrm{Ta}(6.3 \mathrm{keV})$ and ${ }^{83} \mathrm{Kr}(9.4 \mathrm{keV})$ will be possible. This also opens exciting venues for nuclear inelastic scattering using the 6.3 keV Fe K-x-rays in dedicated synchrotron beams. 


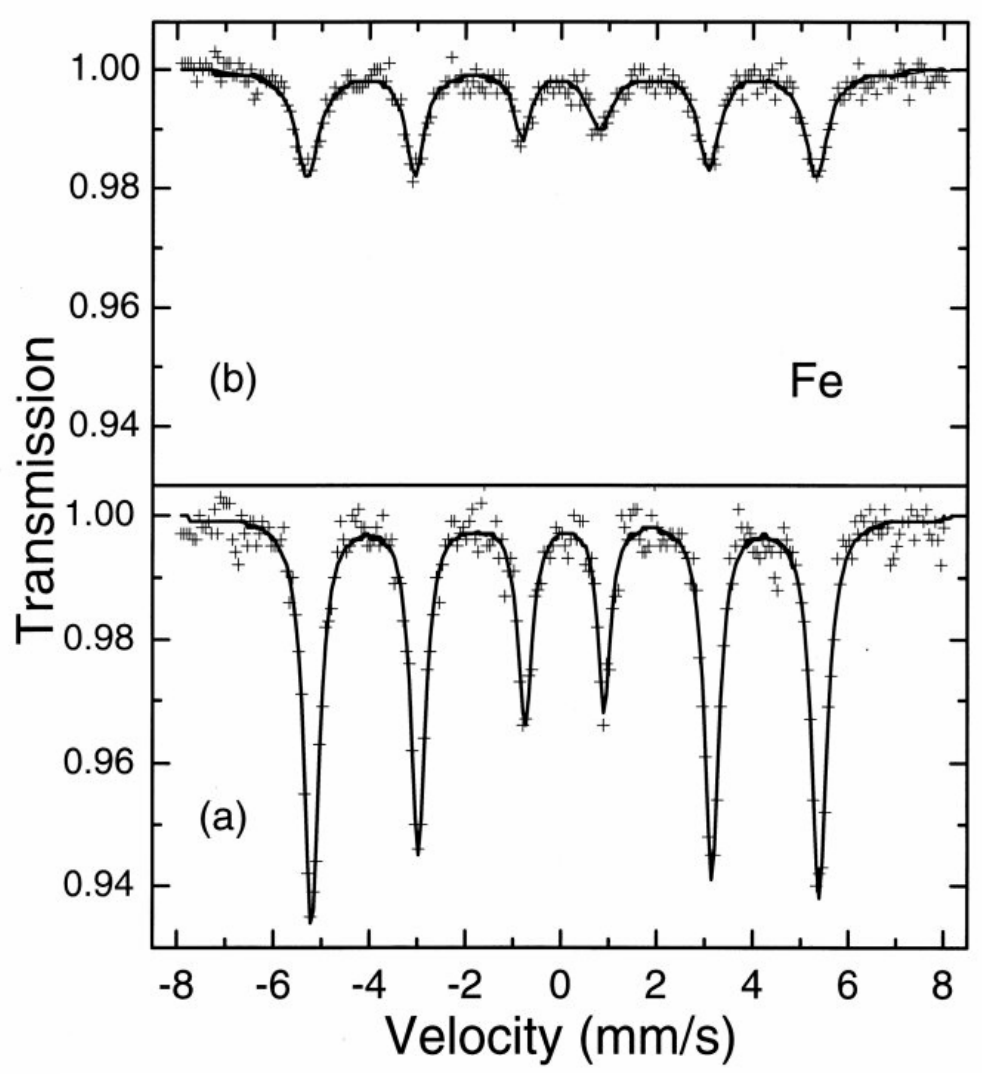

Figure 3 (a) Room temperature MS of $25 \mu \mathrm{m}$ natural iron foil using perforated diamonds, a PPF and a DBP+MA anvil arrangement, in a miniature piston/cylinder DAC. The MS in (b) is for the same arrangement except regular 0.3 carat anvils were used. Pressures $<1 \mathrm{GPa}$.

Acknowledgement: This research was supported in part by the BSF grant 1998003 and by Israeli Science Foundation grant 36/00.

\section{References:}

* email: rdt@lanl.gov

[1] A. Jayaraman, Rev. Mod. Phys. 55 (1983) 65.

[2] H. K Mao and R. Hemley, Rev. Mineralogy 37 (1998) 4.

[3] W. A. Bassett, A. J. Anderson, R. A. Mayanovic, and I. M. Chou, Chemical Geology, 167 (2000) 3, and Z. Kristallog. 215 (2000) 711.

[4] M. P. Pasternak and R. D. Taylor in Mössbauer Spectroscopy Applied to Magnetism and Materials Science, Vol. 2, G. Long and F. Grandjean, eds. (Plenum Press, New York 1996) Chapter 8, p167ff.

[5] A. Dadashev, M. P. Pasternak, G. Kh. Rozenberg,' and R. D. Taylor, Rev. Sci. Instr. 72 (2001) 2633.
*T1/34-01-ICAMEz2
LA-UR-01-6194 